Ann. Biol. anim. Bioch. Biophys., I963, 3 (I),2I-3I

\title{
VARIATIONS GENETIQUES DES POPULATIONS FOLLICULAIRES DANS LES OVAIRES DE RATTES IMPUBÈRES
}

\author{
P. MAULÉON et K. HANUMANTHA RAO \\ avec la collaboration technique de Jaquelıne Barbat, Françoise Tharel, et \\ Marie-Madeleine de RevIERS \\ Station centrale de Physiologie animale, \\ Centre national de Recherches zootechniques, Jouy-en-Josas (Seine-et-Oise)
}

\section{SOMMAIRE}

Sur les ovaires de Rattes Wistar immatures appartenant à trois souches consanguines ayant des sensibilités différentes à l'hormone gonadotrope FSH, une analyse de la population folliculaire a été faite.

Le nombre de follicules prïmordiaux est significativement différent entre les trois souches et à l'intérieur d'une même souche entre lignées maternelles; la souche la plus sensible à FSH est non seulement celle qui contient le plus de follicules de Graaf et de follicules en croissance, mais aussi celle qui possède le stock de follicules primordiaux le plus élevé.

\section{INTRODUCTION}

De nombreux laboratoires s'occupant de dosages biologiques ont signalé des différences de sensibilité aux hormones gonadotropes entre des Rats de races SpragueDawley et Wistar (HAMBURGER, I950 ; CourRIER et $a l$., I96I), et entre animaux de même race provenant d'élevages différents. Enfin à l'intérieur d'un même élevage, la sensibilité d'une colonie évolue au cours des années, comme le signale le State Serum Institute de Copenhague.

Etudiant des variations de sensibilité à 1'hormone folliculo-stimulante entre I7 souches consanguines de Rats, FAVE et al. (I962) ont trouvé des différences très significatives de réponses pondérales entre trois d'entre elles, les souches 03. 04 et 12 .

Ces différences de sensibilité hormonale peuvent s'expliquer soit par la qualité spécifique de la propre sécrétion gonadotrope de l'animal, soit par une variation de la possibilité de réponse de l'effecteur c'est-à-dire des follicules ovariens. 
Dans notre étude, nous avons cherché si cette deuxième hypothèse était valable.

Seule une analyse quantitative précise pouvait nous permettre de saisir les variations de la population folliculaire, aussi est-ce dans cette direction que nous avons orienté notre travail.

\section{MATÉRIEL, ET MÉTHODES}

\section{Matériel animal et préparations hormonales}

Les Rats Wistar utilisés proviennent de 3 couples 03 , o4 et 12 appartenant au départ à 3 souches consanguines. A partir de chacun de ces couples a été développée une colonie fermée dans laquelle un taux élevé de consanguinité a été maintenu.

Les ovaires étudiés ont été prélevés sur les animaux âgés de 27 jours, ayant reçu soit du sérum physiologique, soit $20 \mathrm{UI}$ de $\mathrm{HCG}, 20 \mathrm{UI}$ de $\mathrm{HCG}+500 \mu \mathrm{g}$ de FSH, $20 \mathrm{UI}$ de $\mathrm{HCG}+\mathrm{r} 000 \mu \mathrm{g}$ de FSH (FSH d'hypophyse de Mouton préparé selon la méthode de Jutisz et al., 1960) en 6 injections comme il est habituel dans la technique de dosage de FSH selon STEELMAN et Ponlex (I953).

Enfin, une nourriture identique a été distribuée ad libitum à tous les animaux et la colonie a été soumise à un rythme lumineux invariable de I 4 heures de lumière et Io heures d'obscurité pendant toute l'année.

\section{Techniques histologiques de complage et de classification des follicules}

Les ovaires fixés au Bouin Hollande ont été coupés à ro $\mu$ d'épaisseur, montés en série et colorés par le trichrome de Masson. Les follicules ont été comptés et classés en trois groupes caractérisés par la plus grande surface circulaire ou ellipsoïdale $S$ déterminée par la section du follicule et limités par l'extérieur de la couche folliculeuse ou la couche la plus externe de la granulosa. Seuls les follicules dont le noyau de l'ovocyte est présent dans la coupe sont comptés et mesurés (MAULEON, 1963).

Les 3 catégories de follicules considérées sont :

Groupe I : les follicules primordiaux pour lesquels $S$ est inférieur à I $585 \mu^{2}$ et qui correspondent aux follicules ne possédant qu'une seule souche de cellules folliculeuses aplaties ou cuboïdes.

Groupe II : les follicules en croissance, c'est-à-dire ceux qui ont plus d'une couche de cellules folliculeuses et pour lesquels $S$ est inférieur à ${ }_{1} 5^{8} 5^{00} \mu^{2}$. L'antrum apparaît pour une taille de follicule correspondant à $S=10000 \mu^{2}$.

Ces deux populations se retrouvent chez tous les animaux non traités.

Groupe III : les follicules de Graaf pour lesquels la plus grande surface de coupe $\mathrm{S}$ est supérieure à $158500 \mu^{2}$. Ceux-ci ne sont présents que chez les animaux traités.

Ces trois catégories apparaissent nettement définies sur les courbes de fréquence des populations de follicules ovariens (graphiques I, 2, 3 et 4).

Le mode d'échantillonnage et les corrections de comptage sont propres à chacune de ces catégories (MAULÉON, 1963).

\section{RÉSULTATS}

\section{A. - DIFFÉrences de RÉponse pondÉRALE a FSH DES OVAIRES DES 3 SOUCHES DE RAT}

Les courbes de réponse en fonction du logarithme de la dose de FSH Armour sont des droites pour des doses comprise entre :

$60 \mu \mathrm{g}$ et $240 \mu \mathrm{g}$ pour les Rats de la souche 04

$40 \mu \mathrm{g}$ et $360 \mu \mathrm{g}$ pour ceux de la souche 03

$75 \mu \mathrm{g}$ et $600 u \mathrm{~g}$ pour ceux de la souche I2. 


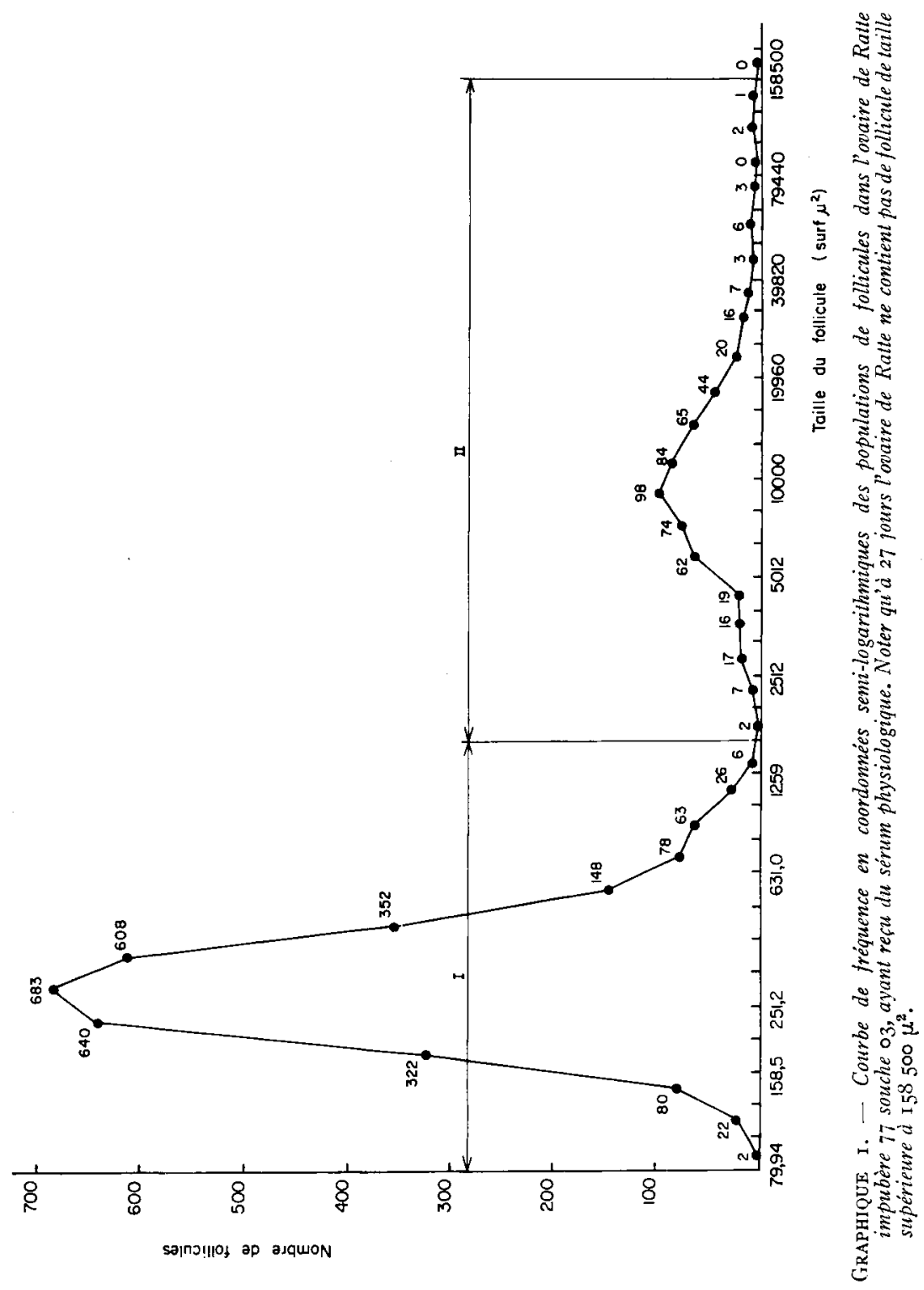


L'analyse de variance montre que dans ces limites la réponse au logarithme de la dose ne s'écarte pas de façon très hautement significative d'une ligne droite.

Surr le graphique 5, nous avons porté les intervalles de confiance $(\mathrm{P}<0,05)$ correspondant pour chaque droite aux différentes valeurs $\mathrm{Y}$ des poids ovariens. Il y apparait clairement que la sensibilité ovarienne à FSH des Rats de la souche I2 est significativement plus faible que celle des animaux des deux autres souches.

Dans le dosage considéré on peut se demander si la réponse des animaux de la souche 04 est plus forte que celle des Rats de souche 03. La comparaison des réponses faites sur les trois doses de FSH les plus fortes pour chacune des 2 souches montre que les droites de réponses sont parfaitement parallèles et que la sensibilité ovarienne des Rats de la souche 04 est en moyenne $\mathrm{I}, 36$ fois plus grande que chez ceux de la souche 03 ; mais nous devons dire que cette différence de sensibilité varie entre $\mathrm{I}, 02$ et $\mathrm{I}, 78(\mathrm{P}<0,05)$ (Emmens, I948).

Les réponses pondérales à l'hormone folliculo-stimulante préparée selon la méthode de Jutisz, des ovaires de Rattes des 3 soutches font également apparaître ces différences de sensibilité (tableau I).

L'ensemble de ces résultats permet dono de dire que la sensibilité ovarienne à FSH des 3 souches de Rat décrô̂t significativement dans l'ordre 04, 03, I2.

TABLEAU I

Variations du poids des ovaires de 3 souches de Rats aprés injection de FSH

\begin{tabular}{|c|c|c|c|c|}
\hline$N^{0}$ de la souche & Traitement hormonal & Nombre d'animaux & $\begin{array}{c}\text { Poids moyen } \\
\text { des ovaires } \\
\overline{\mathrm{X}} \text { en } \mathrm{mg}\end{array}$ & $\begin{array}{c}\text { Erreur standard } \\
\qquad \mathrm{S}_{\overline{\mathbf{x}}}\end{array}$ \\
\hline 04 & $\begin{array}{l}\text { sérum physiologique ....... } \\
\text { HCG ................... } \\
500 \mu \mathrm{FSH} \ldots \ldots \ldots \ldots \ldots \\
1000 \mu \mathrm{g} \text { FSH } \ldots \ldots \ldots \ldots\end{array}$ & $\begin{array}{r}8 \\
5 \\
13 \\
20\end{array}$ & $\begin{array}{r}16,5 \\
30,7 \\
138,7 \\
185,7\end{array}$ & $\begin{array}{r}0,1^{\prime} \\
3,12 \\
13,91 \\
8,15\end{array}$ \\
\hline 03 & $\begin{array}{l}\text { sérum physiologique ...... } \\
\text { IICG ................ } \\
500 \mu \mathrm{g} \text { FSH } \ldots \ldots \ldots \ldots \\
1000 \mu \mathrm{g} \text { FSH } \ldots \ldots \ldots \ldots\end{array}$ & $\begin{array}{r}5 \\
17 \\
6 \\
7\end{array}$ & $\begin{array}{r}17,9 \\
39,7 \\
87,6 \\
151,5\end{array}$ & $\begin{array}{r}0,79 \\
1,52 \\
4,76 \\
18,59\end{array}$ \\
\hline 12 & $\begin{array}{l}\text { sérum physiologique } \ldots \ldots \\
\text { HCG . . . . . . . . . . } \\
500 \mu \mathrm{gSH} \\
1000 \mu \mathrm{g} \text { FSH } \ldots \ldots \ldots \ldots\end{array}$ & $\begin{array}{r}11 \\
8 \\
20 \\
18\end{array}$ & $\begin{array}{l}13,6 \\
28,2 \\
38,8 \\
6-1,7\end{array}$ & $\begin{array}{l}0,95 \\
2,83 \\
2,67 \\
4,91\end{array}$ \\
\hline
\end{tabular}

B. - Différences De Structure ovarienNe entre les 3 souches

I. Analyse de la population de follicules primordiaux

a) Variation du nombre de follicules primordiaux entre les 3 souches.

Les valeurs moyennes trouvées pour les nombres observés de follicules primordiaux sont rapportés dans le tableau 2. 


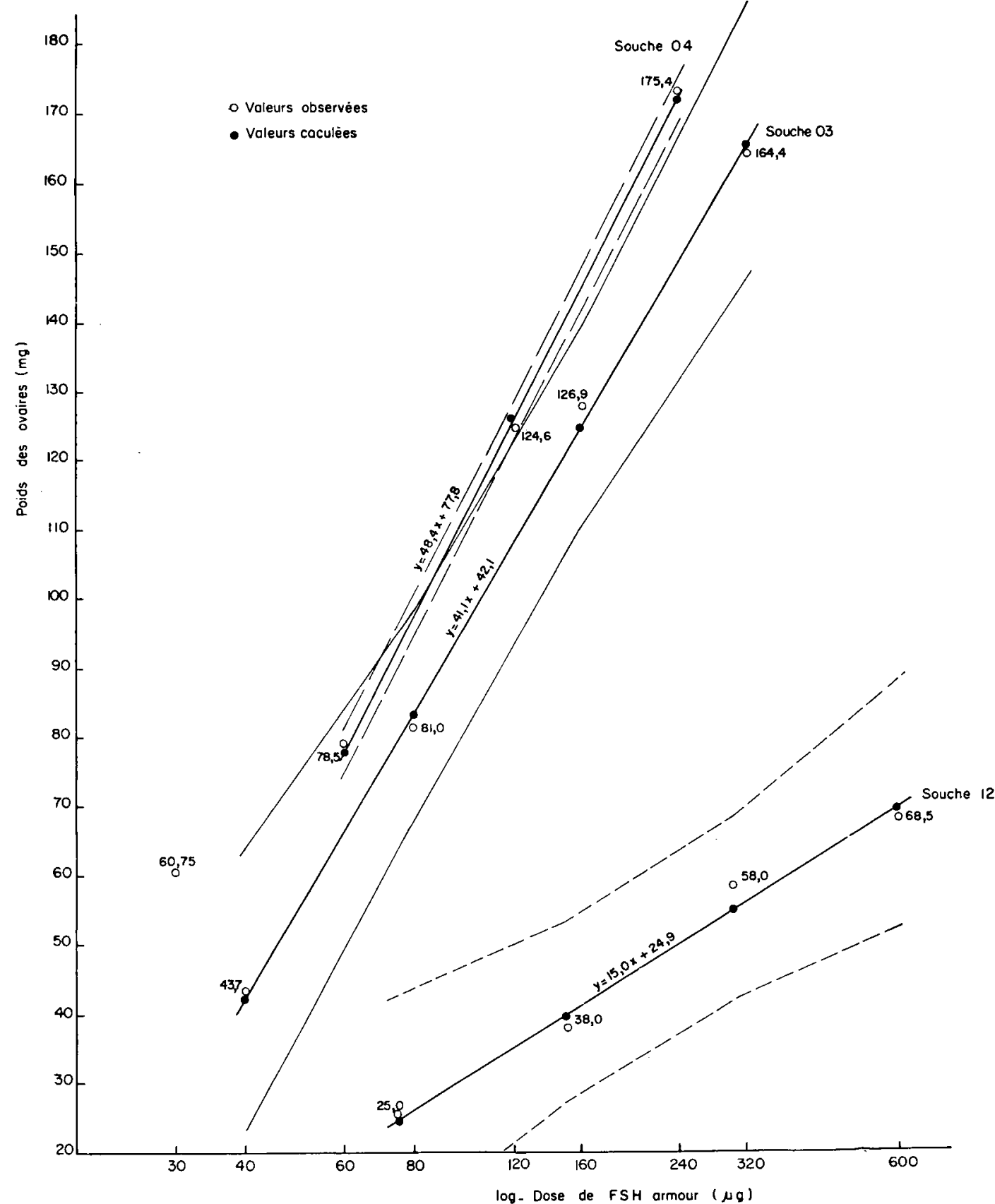

log. Dose de FSH armour $(\mu q)$

GRaPhique 5. - Droites de réponse du poids des ovaires de Rattes impubères à l'hormone folliculostimulante (Armour) selon la méthode STEELMan et POHLEY

- rattes de la souche $04: \mathrm{Y}=48,4 \mathrm{X}+77,8$ (entre $60 \mu \mathrm{g}$ et $240 \mu \mathrm{g}$ )

- rattes de la souche $03: Y=41,1 X+42, I$ (entre $40 \mu \mathrm{g}$ et $320 \mu \mathrm{g}$ )

- rattes de la souche I2 : $Y=I 5,0 X+24,9$ (entre $75 \mu \mathrm{g}$ et $600 \mu \mathrm{g}$ )

$\mathrm{Y}=$ poids des deux ovaires (nombre de rats utilisés pour chaque valeur de $Y: 6$ )

$\mathrm{X}=\log _{2}$ dose considérée/dose minimum. 
TABI,EAU 2

Variations du nombre de follicules primordiaux entre 3 souches de Rat

\begin{tabular}{|c|c|c|c|c|}
\hline No de la souche & Nombre d'animaux & $\begin{array}{l}\text { Nbre inoyen de fol- } \\
\text { licules primordiaux } \\
\text { (dans les } 2 \text { ovaires) }\end{array}$ & $\begin{array}{l}\text { Erreur standard } \\
\qquad \mathrm{S}_{\overline{\mathbf{x}}}\end{array}$ & $\begin{array}{l}\text { Int. confiance } \\
\overline{\mathrm{X}}-t_{0,05} S_{\overline{\mathrm{x}}}<\mu \\
<\overline{\mathrm{X}}+t_{0,05} \mathrm{~s}_{\overline{\mathrm{x}}}\end{array}$ \\
\hline $0_{k}$ & 15 & $1 / 5006$ & 810 & $12269-15743$ \\
\hline 03 & 19 & 9125 & 280 & $8537-9713$ \\
\hline 02 & 28 & 7645 & 403 & $6819-8471$ \\
\hline
\end{tabular}

Entre les souches 04 , et 03,04 et I2, il n'y a aucune superposition des intervalles de confiance; on peut donc dire que le nombre de follicules primordiaux des ovaires des Rattes de la souche 04 est significativement plus élevé que celui des ovaires des Rattes des souches 03 et I2. Les différences entre les souches 03 et I2 bien que n'étant pas aussi nettes, sont cependant hautement significatives $(t=2,72$, $d l=4, \mathrm{P}<\mathrm{O}, \mathrm{OI})$. Il convient de noter également que les 3 souches présentent une grande hétérogénéité en ce qui concerne les variances du caractère nombre de follicules primordiaux ainsi que l'indique le test de BARTLETT $\left(\mathrm{X}^{2}\right.$ corrigé $=\mathbf{1 2 , 8 7}$, calcul selon SNEDECOR, I956) ; la souche la plus homogène est la souche 03.

b) Variations du nombre de follicules primordiaux à l'intérieur de la souche $\mathbf{1} 2$ entre 4 lignées maternelles.

Nous avons cherché si la forte variabilité du caractère "nombre de follicules primordiaux " n'était pas liée à des différences entre lignées maternelles, à l'intérieur d'une souche.

TABLEAU 3

Variations du nombre de follicules primordiaux entre lignées maternelles à l'intérieur de la souche $\mathrm{I} 2$

\begin{tabular}{|c|c|c|c|}
\hline$N^{0}$ de la lignée & Nombre d'animaux & $\begin{array}{c}\text { Nbre moyen de fol- } \\
\text { licules primordiaux } \\
\text { dans les } 2 \text { ovaires } \\
\overline{\mathrm{X}}\end{array}$ & $\begin{array}{l}\text { Erreur standard } \\
\qquad \mathrm{S}_{\overline{\mathbf{x}}}\end{array}$ \\
\hline$w \ldots \ldots \ldots \ldots \ldots \ldots \ldots \ldots$ & 6 & 10325 & 453 \\
\hline 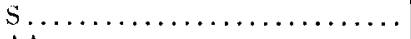 & 4 & 8710 & $109^{\prime}$ \\
\hline $\mathrm{AA} \ldots \ldots \ldots \ldots \ldots \ldots \ldots \ldots \ldots \ldots \ldots$ & 4 & 6756 & 591 \\
\hline $\mathrm{AD}^{2} \ldots \ldots \ldots \ldots \ldots \ldots \ldots \ldots \ldots \ldots$ & 3 & 4819 & 534 \\
\hline
\end{tabular}

L,e test de BARTLETT montre que les variances du nombre observé de follicules primordiaux entre les lignées maternelles de la souche $\mathrm{x} 2$ sont homogènes $\left(\mathrm{X}^{2}\right.$ corrigé $=5,35$ pour $f=3, \mathrm{X}_{0,05}^{2}=7,8 \mathrm{I}$ ). Nous donnons dans le tableau ci-dessous les résultats de l'analyse de variance. 


\begin{tabular}{|c|c|c|c|}
\hline Source de variation à l'intérieur de la souche 12 & Degré de liberté & Carré moyen & $\mathrm{F}$ \\
\hline Variance totale & 16 & & \\
\hline Variance entre lignées maternelles .......... & 3 & 19984283 & $7,65^{* *}$ \\
\hline Variance de l'erreur.................. & 13 & 2609023 & $\mathrm{P}<0,01$ \\
\hline
\end{tabular}

Nous voyons que la variance à l'intérieur de la souche i2 dépend essentiellement de celle entre lignées maternelles ; les différences entre ces trois lignées ne sont pas dues au hasard. Comme il ne s'agit pas d'une expérience planifiée, nous avons utilisé pour comparer individuellement ces moyennes le test de KEULS où chaque différence est comparée à son propre $\mathrm{D}=\mathrm{Q} \cdot \mathrm{S}_{\overline{\mathrm{X}}}$ présenté dans le tableau ci-dessous entre parenthèses.

\begin{tabular}{|c|c|c|c|c|}
\hline Lignée maternelle & $\mathrm{X}$ & $\bar{X}-4819$ & $\bar{X} \ldots 6756$ & $\bar{X}-8710$ \\
\hline 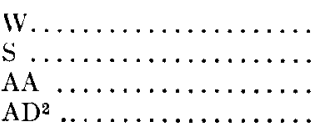 & $\begin{array}{r}10325 \\
8710 \\
6756 \\
4819\end{array}$ & $\begin{array}{l}5506 *(3349) \\
3891 *(3010) \\
1937 \quad(2469)\end{array}$ & $\begin{array}{l}3569^{*}\left(\begin{array}{ll}3 & 010\end{array}\right) \\
195 \prime\end{array}$ & $1615(2 / 469)$ \\
\hline
\end{tabular}

$$
\mathrm{S}_{\overline{\mathrm{x}}}=\sqrt{\frac{\mathrm{s}^{2}}{\mathrm{n}}}=807 \text { et } d . l .=13
$$

La lignée $\mathrm{W}$ est donc très différente des lignées maternelles $\mathrm{AA}$ et $\mathrm{AD}^{2}$; de même la lignée $\mathrm{S}$ par rapport à la lignée $\mathrm{AD}^{2}$. Les autres comparaisons ne permettent pas de conclure dans le sens d'une différence. Cette hétérogénéité des souches indique la nécessité de poursuivre une sélection pour ce caractère sur les lignées maternelles car au départ elles n'avaient pas été sélectionnées dans ce sens.

c) Corrélation entre le poids des ovaires, après une injection de FSH, et le nombre de follicules primordiaux.

Nous avons calculé le coefficient de corrélation entre les deux variables : poids des deux ovaires après une injection de FSH et nombre de follicules primordiaux contenus dans ces mêmes ovaires.

Pour les ovaires ayant reçu $500 \mu \mathrm{g}$ de FSH, le coefficient $r=+0,49$ est significatif à $5 \mathrm{p}$. Ioo $\left(r_{0,05}=0,482\right.$ pour $d . l$. $\left.=I 5\right)$; par contre, pour les ovaires ayant reçu I $000 \mu \mathrm{kg}$ de FSH, ce coefficient de corrélation $r=+0,47$ n'est pas significatif $\left(r_{0,05}=0,576\right.$ pour $\left.d . l .=9\right)$. Ce résultat ne saurait être étonnant, puisque I $000 \mu g$ constituent une dose pour laquelle la réponse ovarienne est quasi maximale.

\section{Analyse de la population des follicules en croissance et des follicules de Graaf}

Cette analyse n'a été faite que sur 3 ovaires, un pour chaque souche et il n'y a pas là un nombre suffisant de résultats pour qu'une analyse statistique soit pos- 


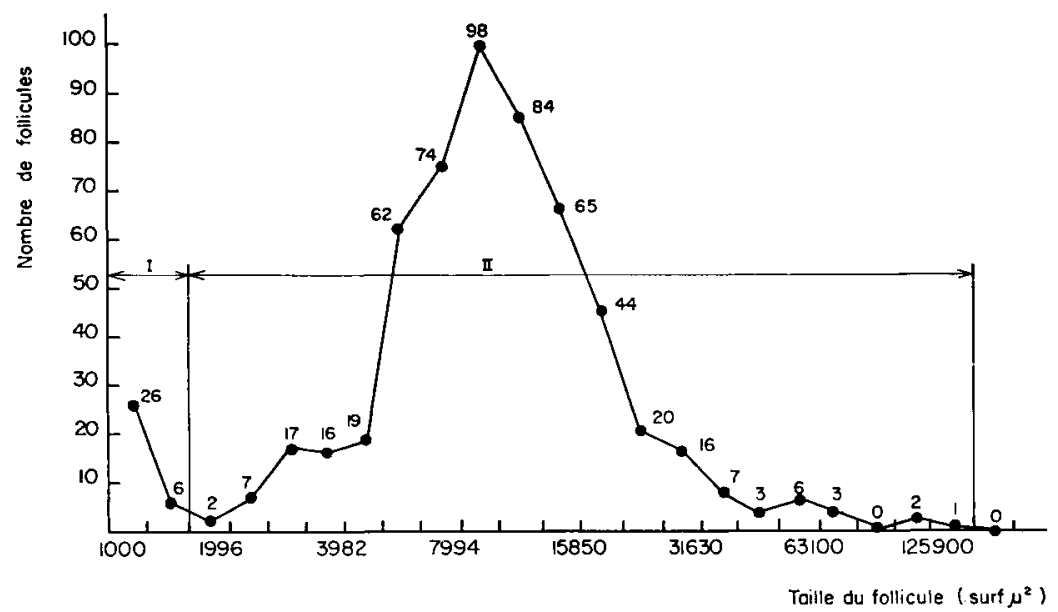

2 - Dans l'ovaire de Ratte impubère 77 souche oz, ayant reçu du résum physiologique (absence de follicule de taille $S>158500 \mu^{2}$

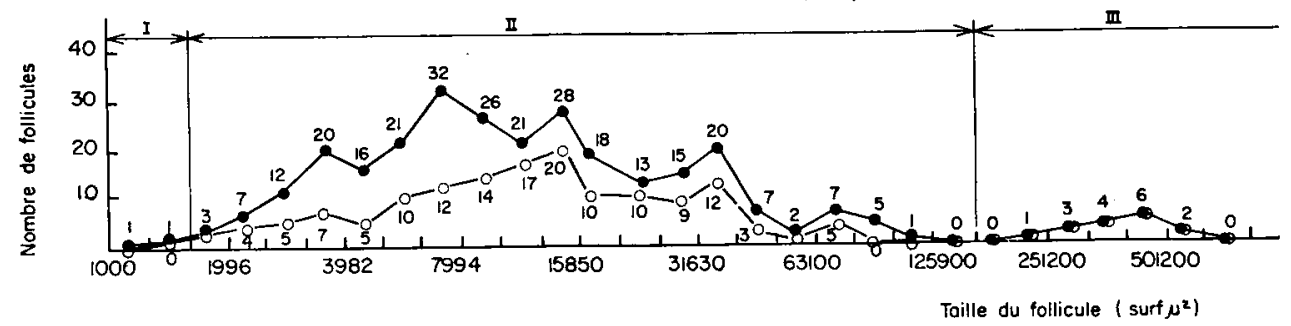

3 - Dans l'ovaire de Ratte impubère 178 souche I 2 , ayant reçu 20 UI de HCG + $500 \mu \mathrm{g}$ de FSH

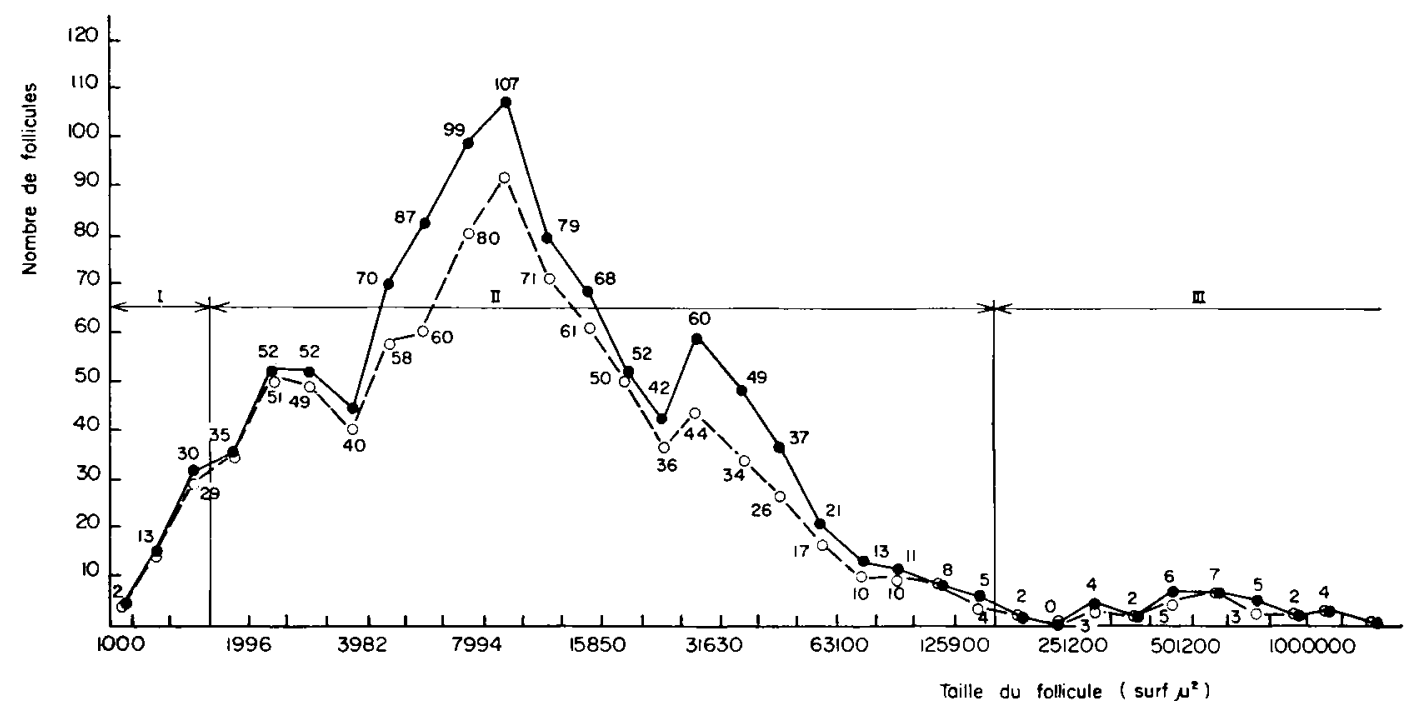

4 - Dans l'ovaire de Ratte impubère 175 souche 04, ayant reçu 20 UI de HCG + $500 \mu g$ de FSII

GRAPHIQUES 2-3-4. - Courbes de fréquences en coordonnées semi-logarithmiques des populations de follicules en croissance et de follicules de Graaf

en trait plein : courbe de fréquence correspondant aux follicules normaux et atrétiques en pointillé : celle correspondant aux follicules normaux. 
sible. Les courbes de distribution des populations folliculaires obtenues sont cependant si nettes dans leurs différences qu'une série de constatations intéressantes peut être tirée.

Comme pour la population de follicules primordiaux, le nombre des follicules en croissance est plus élevé dans la souche 04 , souche la plus sensible à FSH et le moins élevé dans la souche I2, souche la moins sensible à FSH. Les follicules de Graaf, qui mesurent directement la réponse des ovaires à FSH et sont un des éléments déterminant l'augmentation du poids de l'ovaire, sont aussi les plus nombreux dans la souche la plus sensible (graphiques 2,3 et 4 ).

La population des follicules d'un ovaire semble composée de 3 catégories liées par des rapports constants établissant une hiérarchie folliculaire (tableau 4 ).

\section{TABI,EAU 4}

Comparaison des différentes catégories de follicules ovariens entre les 3 souches de Rals

\begin{tabular}{|c|c|c|c|}
\hline Souche & $\begin{array}{l}\text { Nbre corrigé de foll. } \\
\text { primordiaux } S=1585 \mu^{2}\end{array}$ & $\begin{array}{l}\text { Nbre corrigé de foll. en } \\
\text { croissance } 1585 \mu^{2}<\mathrm{S}< \\
158500 \mu^{2}\end{array}$ & $\frac{\text { nbre de foll. en croissance }}{\text { nbre de foll. primordiaux }}$ \\
\hline $\begin{array}{l}04(1) \\
03 \\
12(1)\end{array}$ & $\begin{array}{l}6760 \\
3368 \\
2819\end{array}$ & $\begin{array}{r}1120 \\
547 \\
\geq 75\end{array}$ & $\begin{array}{r}16,5 \\
16,2 \\
9,3\end{array}$ \\
\hline Souche & $\begin{array}{l}\text { Nbre corrige de foll. en } \\
\text { croissance } 1585 \mu^{2}<\mathrm{S}< \\
158500 \mu^{2}\end{array}$ & $\begin{array}{l}\text { Nbre corrigé de foll. de } \\
\text { Graaf } \mathrm{S}<158500 \mu^{2}\end{array}$ & $\frac{\text { nbre de foll. de Graaf }}{\text { nbre defoll. en croissance }}$ \\
\hline $\begin{array}{l}0 \div(1) \\
12(1)\end{array}$ & $\begin{array}{r}1120 \\
275\end{array}$ & $\begin{array}{l}30 \\
16\end{array}$ & $\begin{array}{l}2,7 \\
5,8\end{array}$ \\
\hline
\end{tabular}

(1) Animaux ayant reçu $500 \mu g$ de FSH.

\section{DISCUSSION}

Nous pensons que deux séries de conclusions importantes peuvent être déduites de nos résultats.

I. Le nombre de follicules primordiaux présents dans les ovaires varie entre souches et même entre lignées maternelles. JONES et KROHN (I96I) ont rapporté un résultat analogue chez la Souris; sur 3 souches de Souris CBA, A, R III, et une souche $\mathrm{CBA} \times \mathrm{A}$, les nombres moyens d'ovocytes sont, le jour de la naissance, respectivement de 9775 , I4 365, I5 300 et Io 785 . I1 a été également montré que le nombre d'ovarioles dans les ovaires de Drosopnhile est un excellent critère discriminatoire des 2 populations française et japonaise de Drosophile qui possèdent chacune un patrimoine héréditaire distinct (MELOU, I96I). 
Il est donc vraisemblable que le nombre de follicules primordiaux dont dispose une femelle au début de sa vie sexuelle est un caractère dépendant d'un facteur génétique, la nature de la transmission de ce caractère restant à préciser.

2. Les variations dans le nombre de follicules primordiaux présents dans un ovaire entraînent une variation de sensibilité à FSH.

I'injection de l'hormone FSH entraîne la différenciation de follicules de Graaf qui n'existent pas dans l'ovaire de Ratte immature à l'âge de 27 jours. Il est classique de dire que le follicule ne répond aux hormones gonadotropes qu'à partir du moment où il a acquis son antrum. Aussi est-il normal de trouver une relation entre nombre de follicules de Graaf et nombre de follicules en croissance. Mussertr et PARRotT (I96I) trouvent une relation identique dans les ovaires de Souris qu'ils greffent orthotopiquement.

Par contre il est moins habituel de dire que le nombre de follicules de Graaf formés après une injection de FSH dépend du nombre de follicules primordiaux présents dans cet ovaire. Si cette relation existe, le nombre d'ovulations et la qualité même des cufs pourraient dépendre du nombre de follicules primordiaux. Il s'agit là d'un problème fondamental. La fertilité des animaux dépendrait alors elle-même du nombre d'ovocytes, bien que les opinions sur ce sujet soient contradictoires (Jones et KroHN, I96I ; INGRAM, I958).

Si ces faits se confirment, l'ancienne affirmation à priori, que le nombre d'ovocytes présents dans un ovaire est largement suffisant pour la vie de l'animal perdrait beaucoup de sa valeur et la notion de production quantitative de gamètes femelles prendrait autant de force que celle admise maintenant pour le mâle.

\section{CONCLUSION}

Il existe entre les souches de Rats de même race des variations dans les populations de différents follicules ovariens (follicules primordiaux, follicules en croissance et follicules de Graaf). Ces variations semblent liées à celles de la sensibilité à !'hormone folliculo-stimulante de l'ovaire.

Rę̧ pour publication en novembre 1962.

\section{SUMMARY}

GENETIC VARIATIONS IN THE FOLLICULAR POPULATION OF THE OVARIES OF PREPLBERTAL RAT

Prepubertal rats of 3 inbred strains (04, 03 and I2) show dose/response lines for ovarian weight on $\log$ dose of FSH (Armour) which are significantly different (fig. 5).

A quantitative analysis of the follicular population of these 3 strains has shown that the number of primordial follicles is significantly different between strains, and also within strains between maternal lines (tables 2 and 3 ).

The strain most sensitive to FSH (o4) is that which contains not only largest numbers of growing and developed Graafian follicles, but also the largest number of primordial follicles $(r=$ $+0,45$ ) (fig. $2,3,4$ ). 


\section{RÉFÉRENCES BIBLIOGRAPHIQUES}

Colrrier R., Guillemin R., Jutisz M., Sakiz. li., Ascineme P., ig6r. Présence dans un extrait d'hypothalamus d'une substance qui stimule la sécrétion de l'hormone antehypophysaire de lutéinisation (LH). C. R. Acad. Sci., 253, 922-927.

Emmens C. W., 1948. Principles of biological assay. Chapman and Hall. London.

Fave A., Gillois J., Ortavant R., Poly J., I962. (Communication personnelle).

Hamburger C, i950 in Emmens c. w., Hormone Assay Academic Press inc. New York.

INGRAM D. L., 1958. Fertility and oocyte number after $\mathrm{X}$ irradiation of the ovary. J. Endocrin., 17, $8 \mathrm{I}-90$.

Jones E. C., Kronn P. L., I96r. The relationships between age, number of oocytes and fertility in virgin and multiparous Mice. J. Endocrin., 21, 469-495.

Jutisz M., Hermier C., Colonge A., Courrier R., ig60. Isolement des hormones hypophysaires; purification de l'hormone folliculostimulante de Mouton. C. R. Acal. Sci., 250, 43[-435.

Maulíon P., 1963. Méthode de comptage et de classification des follicules ovariens. Ann. Biol. anim. Bioch. Biophys. (à paraître).

Melou J. P., I96r. Etude du nombre d'ovarioles chez diverses souches françaises et japonaises de Drosophila melanogaster. Ann. Génét., 3, 25-28.

MussetT M. I., Parrott D., I961. Factors affecting the fertility of mice. J. Reprod. Fertil., 2, 80-97 Snedecor, 1956. Statistical methods. The Iowa State University Press. Ames. Iowa. U. S. A.

Steelman S. L., Pohley F. M., I953. Assay of the follicle stimulating hormone based on the augmentation with human chorionic gonadotrophin. Endocrinology, 53, 604-6r6. 